\title{
Safety profiles of current antiangiogenic therapies for metastatic colorectal cancer
}

This article was published in the following Dove Press journal:

OncoTargets and Therapy

16 August 2012

Number of times this article has been viewed

\section{Jyotsna Fuloria}

Ochsner Medical Center, New Orleans, LA, USA
Correspondence: Jyotsna Fuloria

Ochsner Medical Center,

1514 Jefferson Highway

New Orleans, LA, 70I2I USA

$\mathrm{Tel}+\mathrm{I} 5048423910$

Fax + I 5048424533

Email jfuloria@ochsner.org

\begin{abstract}
The biological agents approved for the treatment of patients with metastatic colorectal cancer - bevacizumab, a monoclonal antibody that targets vascular endothelial growth factor A, along with cetuximab and panitumumab, two monoclonal antibodies that target the epidermal growth factor receptor - are associated with a number of adverse events that range in severity from relatively mild to potentially life threatening. Hypertension, thromboembolic events, proteinuria, bleeding, and gastrointestinal perforation have all been associated with bevacizumab, while dermatologic toxicities are common with cetuximab and panitumumab. Hypersensitivity reactions and hypomagnesemia are also a concern with cetuximab and panitumumab. The frequency of these adverse events in randomized clinical trials is reviewed, and recommendations for managing these events in patients undergoing treatment for metastatic colorectal cancer are provided.
\end{abstract}

Keywords: adverse events, antiangiogenic agents, bevacizumab, cetuximab, metastatic colorectal cancer, panitumumab

\section{Introduction}

It has been four decades since Folkman proposed the use of antiangiogenic therapies for cancer. ${ }^{1}$ Angiogenesis, or the formation of new blood vessels, plays a key role in the pathogenesis of cancer and is required for the growth of solid tumors and metastases, ${ }^{1,2}$ with the vascular endothelial growth factor (VEGF) signaling pathway playing a central part in the angiogenesis network..$^{3-6}$ The VEGF family of cytokines includes VEGF-A, VEGF-B, VEGF-C, VEGF-D, and placental growth factor (PlGF). VEGF-A binding to the VEGF receptors (VEGFR-1 and VEGFR-2) activates pro-angiogenic signaling pathways that are associated with increased vascular permeability, mobilization of progenitor endothelial cells, and assembly of vascular networks though endothelial proliferation and migration. ${ }^{7}$ It also promotes cancer cell survival, migration, and invasion. ${ }^{7}$ Overexpression of VEGF-A may also play an early role in the establishment of colorectal cancers. ${ }^{8}$ VEGF-B and PlGF have gained more interest over recent years for their roles in tumor angiogenesis. VEGF-B is critical for the survival of vascular endothelial cells, pericytes, smooth muscle cells, and vascular stem/progenitor cells; ${ }^{9}$ in addition, through VEGFR-1 signaling, VEGF-B may be important in the epithelialmesenchymal transition, which could play a role in tumor progression and metastasis. ${ }^{10}$ Overexpression of PlGF in preclinical studies has been associated with an increase in the number and size of blood vessels, in addition to increased vascular leakiness, suggesting a strong angiogenic effect of PlGF in vivo. ${ }^{11}$ 
Currently, three targeted monoclonal antibodies (mAbs) with antiangiogenic effects are approved for the treatment of metastatic colorectal cancer (mCRC): bevacizumab, cetuximab, and panitumumab. Bevacizumab is a humanized $\mathrm{mAb}$ that targets VEGF-A, ${ }^{12,13}$ and it is approved in the United States and Europe for the treatment of mCRC in combination with fluoropyrimidine-based chemotherapy in both first- and second-line settings. ${ }^{12,14}$ Cetuximab, a chimeric mouse/human $\mathrm{mAb}$, and panitumumab, a fully human $\mathrm{mAb}$, both inhibit the epidermal growth factor receptor (EGFR). ${ }^{15,16}$ EGFR signaling appears to modulate angiogenesis via the upregulation of angiogenic factors, such as VEGF, and inhibition of the EGFR pathway has been shown to inhibit angiogenesis, tumor growth, and metastasis. ${ }^{17}$ Although EGFR is overexpressed in epithelial cancers, including colorectal cancer, its role as a prognostic factor remains controversial. ${ }^{18}$ Both cetuximab and panitumumab are approved in the United States and Europe for the treatment of mCRC in patients with $K R A S$ wild-type, whose tumors express EGFR. ${ }^{15,16}$ Cetuximab is indicated as a monotherapy following failure of both irinotecan- and oxaliplatin-based chemotherapy or in patients intolerant to irinotecan, and in combination with irinotecan in patients refractory to irinotecan-based chemotherapy. ${ }^{15,19}$ In Europe, cetuximab also is indicated for use in combination with oxaliplatin, 5-fluorouracil (5-FU), and leucovorin (FOLFOX4). ${ }^{19}$ Panitumumab is approved as monotherapy in the second-line setting following treatment with fluoropyrimidine-, oxaliplatin-, or irinotecan-containing chemotherapy regimens. In Europe, panitumumab also is indicated for $\mathrm{mCRC}$ in the first-line setting in combination with FOLFOX, and in the second-line setting in combination with leucovorin, 5-FU, and irinotecan (FOLFIRI) following first-line fluoropyrimidine regimens that did not contain irinotecan. ${ }^{16,20,21}$

This review characterizes the safety profiles of bevacizumab, cetuximab, and panitumumab. In addition, recommendations for managing adverse events (AEs) associated with these agents in patients with mCRC are provided.

\section{Adverse events associated with bevacizumab}

In first-line combination trials, the chemotherapy backbone used was irinotecan, bolus 5-FU, and leucovorin (IFL); ${ }^{22}$ bolus 5-FU and leucovorin; $; 3,24$ or oxaliplatin-based chemotherapy (oxaliplatin plus capecitabine [XELOX] or FOLFOX4). ${ }^{25}$ In a phase III trial in the second-line setting, the chemotherapy backbone was FOLFOX $4 .{ }^{26}$
Across all clinical trials, hypertension has been observed more frequently in patients with mCRC treated with bevacizumab in combination with chemotherapy than in those treated with chemotherapy alone. ${ }^{27}$ The incidence of grade 3 or higher hypertension was substantially higher in the bevacizumab treatment groups compared with controls, particularly in the first-line setting (Table 1). ${ }^{22-26}$ Although all-grade hypertension is less frequently described in published mCRC clinical studies, higher incidences have been reported with bevacizumab than in control groups $(11 \%-32 \%$ vs $3 \%-8.3 \%$, respectively) (Table 1$) .{ }^{22-24}$ While $P$ values were not reported in the phase II trials, the difference between the bevacizumab and placebo groups was significant $(P<0.01)$ in the phase III trial. ${ }^{22-24}$ Little guidance currently exists for the management of hypertension in patients with $\mathrm{mCRC}$ who are receiving bevacizumab. ${ }^{28}$ Although it is usually possible to control hypertension with standard antihypertensive medications in most patients, it may be necessary to temporarily or permanently discontinue bevacizumab if hypertension is severe or persistent. ${ }^{28}$ Other AEs associated with bevacizumab treatment include thromboembolism, hemorrhage, and proteinuria. ${ }^{22}$ While malignancy, mobility, chemotherapy, antiangiogenic agents, and comorbidity conditions can affect the risk of developing venous thromboembolisms, bevacizumab increases the risk of arterial thromboembolisms from approximately $1 \%$ to $3 \% .^{12,29-31}$ While one phase II trial of bevacizumab in a first-line setting with mCRC patients reported a greater incidence of thrombotic events in patients receiving bevacizumab than in those taking 5-FU/leucovorin alone (13\%-26\% vs $9 \%$ [ $6 \%-14 \%$ vs $3 \%$ grade $3 / 4]$ ) (Table 1$),{ }^{23}$ a second trial using the same chemotherapy backbone reported no difference between the bevacizumab and placebo arms (18\% for each). ${ }^{24}$ In the latter trial, there were twice as many patients with arterial thrombotic events in the bevacizumab arm than in the placebo arm (10\% vs $5 \%){ }^{24}$ The percentages of patients with deep thrombophlebitis and pulmonary embolus with bevacizumab were $6 \%$ and $3 \%$, respectively; with placebo, they were $9 \%$ and $2 \%$, respectively. ${ }^{24}$ In a phase III trial in the first-line setting, no statistically significant increases were observed in bevacizumab plus IFL versus placebo plus IFL in the incidence of any thrombotic event (19.4\% vs $16.2 \%$ ), deep thrombophlebitis ( $8.9 \%$ vs $6.3 \%$ ), or pulmonary embolus ( $3.6 \%$ vs $5.1 \%) .{ }^{22}$ Using an oxaliplatin-based chemotherapy backbone, thromboembolic events in a phase III trial of bevacizumab in a first-line setting were the most common pre-defined grade 3 or 4 AEs considered potentially related to bevacizumab. ${ }^{25}$ In that trial, 


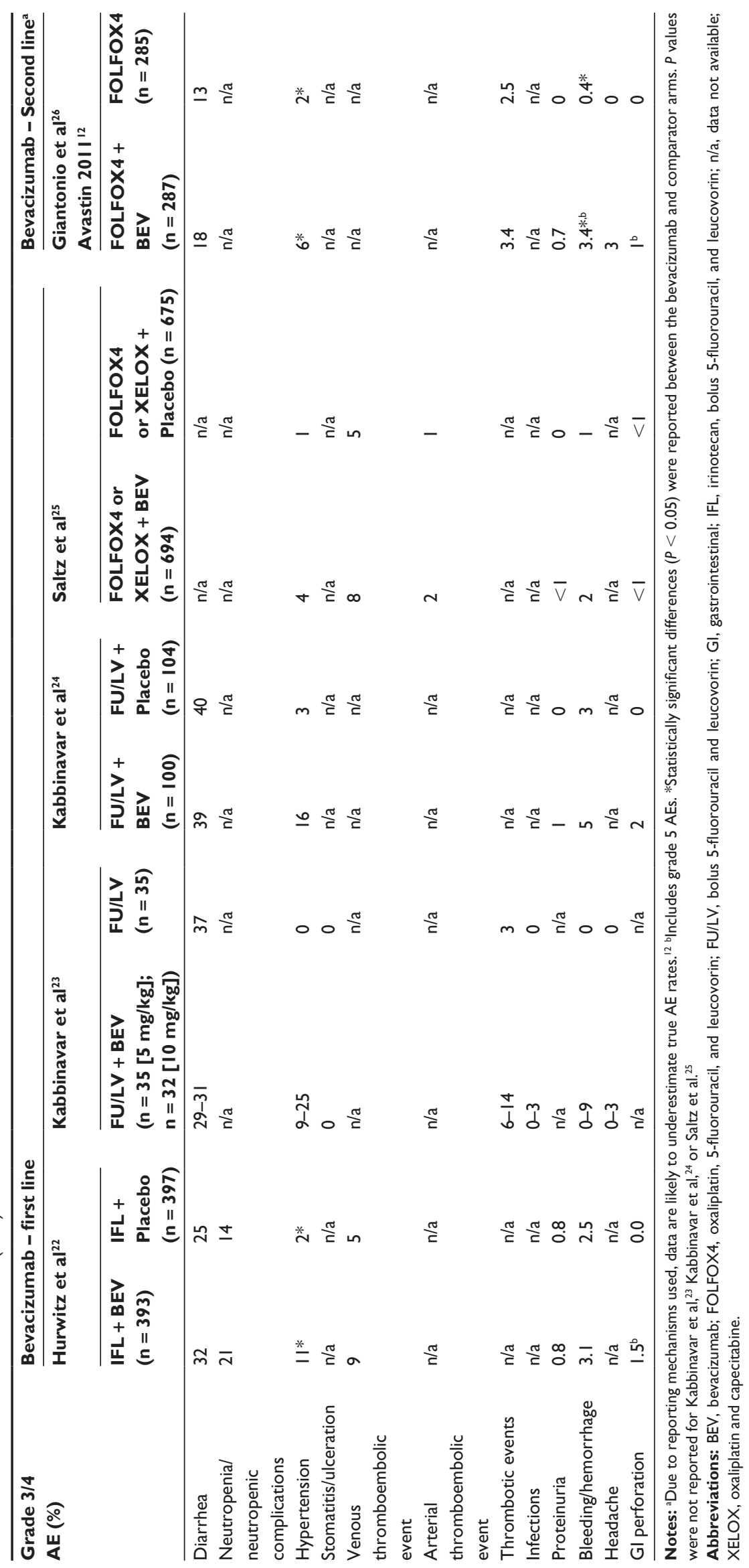


the incidence of grade 3 or 4 venous thromboembolic events was $8 \%$ with bevacizumab versus $5 \%$ with placebo, and the incidence of arterial thromboembolic events was $2 \%$ versus $1 \%$, respectively (Table 1 ). ${ }^{25}$ In the second-line setting, the incidence of grade 3 or 4 thromboembolic events was comparable with bevacizumab plus FOLFOX4 versus FOLFOX4 alone (3.4\% vs $2.5 \% ; P=0.62$; Table 1$).{ }^{26}$ Based on an analysis of data from patients treated with bevacizumab for $\mathrm{mCRC}$, as well as other indications, elderly patients and those with a history of arterial thromboembolism are considered to be at greater risk for arterial thromboembolic events. ${ }^{12,29}$ As the addition of aspirin does not appear to increase the risk for severe bleeding events over that of bevacizumab plus chemotherapy, it has been suggested that the prophylactic use of aspirin may be considered in patients at increased risk of arterial thromboembolic events who have no contraindications. ${ }^{29}$ This was based on pooled analysis of five randomized controlled trials of bevacizumab in patients with metastatic cancers in which aspirin was associated with only a modest increase in bleeding (1.3-fold) in both the bevacizumab and control groups. ${ }^{29}$ Bevacizumab should be discontinued in patients who experience a severe arterial thromboembolic event. ${ }^{12}$

Severe, sometimes fatal bleeding episodes have been reported more frequently in patients with $\mathrm{mCRC}$ who have been treated with bevacizumab in combination with chemotherapy, when compared with those treated with chemotherapy alone. ${ }^{12}$ Grade 3 or 4 gastrointestinal hemorrhage was observed in three patients (9\%) receiving bevacizumab plus 5-FU/leucovorin in one phase II mCRC trial, but it was not clear whether these events were related to treatment. ${ }^{23}$ In a second trial, grade 3 or 4 bleeding events were reported in $5 \%$ of patients receiving bevacizumab plus 5-FU/leucovorin, compared with $3 \%$ in the control group (Table 1). ${ }^{24}$ In phase III trials in the first-line setting, the percentage of patients with grade 3 or 4 bleeding in the bevacizumab group versus the control group was $2 \%-3.1 \%$ versus $1 \%-2.5 \%$, respectively (Table 1 ). ${ }^{22,25}$ In the first trial, the difference was not statistically significant; significance was not reported in the second trial. ${ }^{22,25}$ In the second-line setting, there was a significantly increased incidence of grade 3 or 4 bleeding with bevacizumab plus FOLFOX4, compared with FOLFOX4 alone $(3.4 \%$ vs $0.4 \%, P=0.011)$ (Table 1). ${ }^{26}$ Severe bleeding complications are included in a black box warning in the Prescribing Information. ${ }^{12}$ Bevacizumab should not be given to patients with a recent history of hemoptysis ( $\geq 1$ teaspoon of red blood) or serious hemorrhage. ${ }^{12}$ It has been suggested that minor bleeding (such as epistaxis) may be managed with usual interventions without the discontinuation of bevacizumab. ${ }^{32,33}$ However, based on the clinical situation, major bleeding may require cessation of bevacizumab.

An increased incidence of proteinuria with bevacizumab plus chemotherapy compared with chemotherapy alone has been reported in the first-line setting (19\%-38\% vs 11\%-19\% [1\% vs $0 \%$ grade 3$]) .^{23,24}$ Grade 3 or 4 proteinuria was reported in $<1 \%$ of patients receiving bevacizumab in phase III trials in patients with $\mathrm{mCRC}$, with no significant difference between the bevacizumab and comparator arms in the two trials in which $P$ values were reported (Table 1). ${ }^{22,25,26}$ Regular dipstick analysis of urinary protein is recommended for all patients receiving bevacizumab. ${ }^{12}$ Doses should be temporarily suspended in the event of $\geq 2 \mathrm{~g} / 24$ hours urinary protein, and bevacizumab should be discontinued in patients with nephrotic syndrome. ${ }^{12}$

Gastrointestinal perforation also has been associated with bevacizumab in studies of patients with mCRC. A first-line phase II trial reported gastrointestinal perforation in $2 \%$ of patients with mCRC receiving bevacizumab, with no events observed in the control group (Table 1). ${ }^{24}$ Another first-line phase II trial did not report gastrointestinal perforation (Table 1). ${ }^{23}$ In two phase III studies of first-line bevacizumab, the incidence of perforation with bevacizumab was $1.5 \%$ and $<1 \%$, respectively, compared with $0 \%$ and $<1 \%$ with placebo; three of these events were fatal (two patients receiving bevacizumab and one patient receiving placebo) (Table 1 ). ${ }^{22,25}$ The difference in the incidence of perforation was not statistically significant in the first trial, and $P$ values were not reported in the second trial. $^{22,25}$ Gastrointestinal perforation was also reported in six patients receiving bevacizumab in a randomized, placebocontrolled, phase III trial in the second-line setting (1\%; fatal in two patients) (Table 1). ${ }^{26}$ This complication appeared to be associated with peptic ulcer disease, peritoneal carcinomatosis, recent colonoscopy, and recent colon surgery. ${ }^{22,24}$ A retrospective sub-analysis of patients who participated in a phase III study of bevacizumab in patients with $\mathrm{mCRC}$ found that symptomatic gastrointestinal ulcers are a side effect of treatment and may precede the emergence of perforation. ${ }^{34}$ It is necessary to discontinue bevacizumab in patients with gastrointestinal perforation. ${ }^{12}$

Although wound healing complications can be associated with bevacizumab treatment, given the role of VEGF in that process, reports of grade $3 / 4$ wound healing complications in $\mathrm{mCRC}$ trials were rare $(<1 \%) .{ }^{25}$ It should be taken into account, however, that bevacizumab therapy was not initiated 
until at least 28 days after surgery in clinical trials. ${ }^{12,22-26}$ Wound healing complications that require medical intervention also require discontinuation of bevacizumab. ${ }^{12}$

A higher frequency of grade 3 and 4 neutropenia has been observed with first-line bevacizumab compared with IFL alone ( $21 \%$ vs $14 \%$; Table 1$).{ }^{12}$ The incidence of grade 3 or 4 diarrhea in two large studies has suggested a possible exacerbation of chemotherapy-related diarrhea by bevacizumab - with rates of $32 \%$ versus $25 \%$ in the first-line setting 22 and $18 \%$ versus $13 \%$ in the second-line setting $^{12}$ - reported for bevacizumab plus chemotherapy versus chemotherapy alone, respectively. The difference in the first-line study was not statistically significant, ${ }^{22}$ and $P$ values were not reported for this parameter in the secondline study. ${ }^{12}$ In contrast, two smaller studies reported similar rates between treatment arms (31\%-39\% vs $37 \%-40 \%$; Table 1). ${ }^{23,24} \mathrm{~A}$ similar incidence of stomatitis, an AE also associated with 5-FU/leucovorin, was reported with bevacizumab plus 5-FU/leucovorin versus 5-FU/leucovorin alone in a first-line phase II trial (19\%-23\% vs $17 \%$, respectively) ${ }^{23}$ grade 3 or 4 stomatitis was not observed in this study.

\section{Adverse events associated with cetuximab and panitumumab}

Cetuximab has been investigated in phase II and III trials for the first-line treatment of patients with advanced or mCRC, in combination with XELOX and bevacizumab, ${ }^{35}$ FOLFIRI; $;^{36}$ XELOX $;^{37}$ FOLFOX $4 ;^{38}$ and oxaliplatinfluoropyrimidine combinations (ie, 5-FU or capecitabine). ${ }^{39}$ Second-line cetuximab has been investigated in combination with irinotecan ${ }^{40}$ and as monotherapy with best supportive care. ${ }^{41}$ Panitumumab has been assessed in the first-line setting in combination with bevacizumab plus oxaliplatin- or irinotecan-based chemotherapy, ${ }^{42}$ in the second-line setting in combination with FOLFIRI, ${ }^{43}$ and as monotherapy with best supportive care. ${ }^{44,45}$

Dermatologic toxicities, including papulopustular rash, dry skin, pruritus, and paronychia, are commonly observed with EGFR inhibitors, ${ }^{46}$ and have been positively correlated with clinical response in patients with $\mathrm{mCRC}$ who were treated with the anti-EGFR mAbs cetuximab and panitumumab. ${ }^{44,47}$ In the first-line setting, the incidence of mild to moderate skin AEs was comparable with cetuximab in combination with bevacizumab plus chemotherapy versus bevacizumab plus chemotherapy alone (54\% vs 51\%, respectively). ${ }^{35}$ However, the incidence of grade 3 or 4 skin toxicities was significantly higher with cetuximab ( $38 \%$ vs $19 \% ; P<0.0001$ ), largely due to an increased incidence of grade $3 / 4$

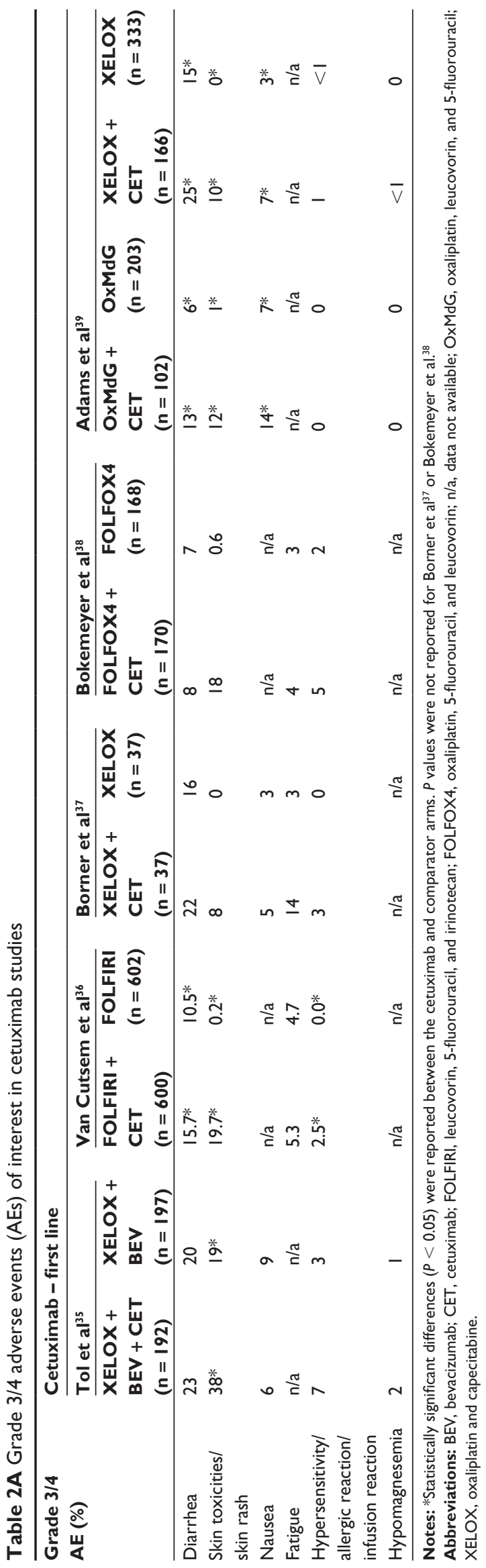


acneiform skin rash $(26 \%$ vs $0.5 \% ; P<0.001)$ (Table $2 \mathrm{~A}) .{ }^{35}$ First-line cetuximab also was associated with an increase in grade 3 or 4 skin toxicities versus the respective control groups when combined with FOLFIRI (all skin toxicities: $19.7 \%$ vs $0.2 \%$; $P<0.001$; acne-like rash: $16.2 \%$ vs $0 \%$; $P<0.001$ ), ${ }^{36}$ XELOX (skin rash: $8 \%$ vs $0 \%$ ), ${ }^{37}$ FOLFOX4 (skin reactions: $18 \%$ vs $0.6 \%$ ), ${ }^{38}$ and oxaliplatin plus 5-FU or capecitabine (skin rash: $10 \%-12 \%$ vs $0 \%-1 \%$; $P<0.001 \pm$ cetuximab) (Table $2 \mathrm{~A}){ }^{39}$

In the second-line setting, acneiform rash occurred in significantly more patients receiving cetuximab plus irinotecan than irinotecan alone $(76.3 \%$ vs $4.9 \%$ [all grade]; $8.2 \%$ vs $0.2 \%$ [grade $3 / 4$ ]; $P<0.05$ for both comparisons) (Table 2B). ${ }^{40}$ Second-line cetuximab monotherapy was associated with a significant increase in grade 3 rash compared with best supportive care alone ( $11.8 \%$ vs $0.4 \%$, respectively; $P<0.001$ ) (Table 2B). ${ }^{41}$

Similar to cetuximab, skin toxicities were frequently reported in trials of panitumumab in first- and second-line settings; a higher incidence of grade 3 or 4 skin toxicity events has been reported with the addition of panitumumab, compared with bevacizumab plus oxaliplatin-based chemotherapy alone (36\% vs 1\%), bevacizumab plus irinotecanbased chemotherapy alone (38\% vs $0 \%$; grade 3 only), FOLFIRI $(32 \%-37 \%$ vs $1 \%-2 \%)$, or best supportive care (14\% vs $0 \%$ ) (Table 3). ${ }^{16,42,43}$ In the case of both cetuximab and panitumumab, topical steroids and antibiotics, systemic steroids, oral antibiotics, or oral retinoids are used to manage skin toxicities. ${ }^{48}$ The recommended management of patients with grade 3 or 4 skin toxicities involves a delay in dosing until symptoms improve; treatment is then resumed at a lower dose. ${ }^{15,16}$

Ocular complications, including conjunctivitis (4\%), ocular hyperemia $(3 \%)$, increased lacrimation $(2 \%)$, and eye/eyelid irritation ( $1 \%$ ), have been reported in patients treated with panitumumab. ${ }^{16}$ In a trial comparing panitumumab with best supportive care alone, ocular toxicities were reported in $15 \%$ and $2 \%$ of patients, respectively ( $<1 \%$ vs $0 \%$ grade 3/4) (Table 3). ${ }^{16}$ Management of ocular complications includes artificial tears, as well as steroid eye drops and topical cyclosporine drops when necessary. ${ }^{48}$ Blepharitis and meibomitis are treated using the application of heated compresses. ${ }^{48}$ Blepharitis also necessitates hygienic care and, in many cases, topical anti-inflammatory treatment. ${ }^{48}$ Severe cases of meibomitis may require systemic treatment with doxycycline. ${ }^{48}$ In general, patients should be referred to an ophthalmologist if symptoms are persistent or severe. ${ }^{48}$

As with all mAbs, hypersensitivity reactions are a concern with cetuximab and panitumumab treatment. Patients may have preexisting immunoglobulin $\mathrm{E}$ antibodies that are reactive against the chimeric antibody cetuximab, thus predisposing them to hypersensitivity reactions. ${ }^{49}$ Infusion reactions have been reported in $15 \%-21 \%$ of patients in clinical trials of cetuximab for various indications $(2 \%-5 \%$ grade $3 / 4) .{ }^{15}$ In trials with mCRC patients, grade 3 or 4 hypersensitivity or infusion-related reaction events were reported in 1\%-7\% of patients receiving cetuximab, compared with $\leq 3 \%$ of patients in the control groups (Table 2A, 2B). ${ }^{35-41}$ Out of the four studies that reported statistical significance, two reported $P<0.001$ for that parameter, and one reported $P=0.088 .^{35,36,41}$ In the fourth study, there was no significant difference; only one patient receiving cetuximab experienced a grade 3 or 4 hypersensitivity reaction. ${ }^{39}$ Infusion reactions have also been reported in clinical trials of the fully human mAb panitumumab, with a frequency of $3 \%(1 \%$ grade $3 / 4) .{ }^{16}$ In patients receiving cetuximab or panitumumab, management of mild-to-moderate infusion reactions includes reduction of the infusion rate. ${ }^{15,16}$ Premedication with diphenhydramine

Table 2B Grade 3/4 adverse events (AEs) of interest in cetuximab studies

\begin{tabular}{|c|c|c|c|c|}
\hline \multirow[t]{3}{*}{ Grade 3/4 AE (\%) } & \multicolumn{4}{|c|}{ Cetuximab - second line } \\
\hline & \multicolumn{2}{|l|}{ Sobrero et $\mathrm{al}^{40}$} & \multicolumn{2}{|l|}{ Jonker et $\mathbf{a l}^{4 !}$} \\
\hline & $\begin{array}{l}\text { Irinotecan + CET } \\
(n=638)\end{array}$ & $\begin{array}{l}\text { Irinotecan } \\
(\mathrm{n}=629)\end{array}$ & $\begin{array}{l}\text { BSC + CET } \\
(n=288)\end{array}$ & $\begin{array}{l}\text { BSC } \\
(n=274)\end{array}$ \\
\hline Diarrhea & 28.4 & 15.7 & $\mathrm{n} / \mathrm{a}$ & $\mathrm{n} / \mathrm{a}$ \\
\hline Skin toxicities/skin rash & 8.2 & 0.2 & $11.8^{*}$ & $0.4^{*}$ \\
\hline Nausea & 4.4 & 4.3 & 5.6 & 5.5 \\
\hline Fatigue & 7.7 & 3.3 & 33.0 & 25.9 \\
\hline $\begin{array}{l}\text { Hypersensitivity/allergic } \\
\text { reaction/infusion reaction }\end{array}$ & 1.4 & 0.8 & $4.5^{*}$ & $0^{*}$ \\
\hline Hypomagnesemia & 3.3 & 0.4 & $5.8^{*}$ & $0 *$ \\
\hline
\end{tabular}

Notes: *Statistically significant differences $(P<0.05)$ were reported between the cetuximab and comparator arms. $P$ values were not reported for Sobrero et al. ${ }^{40}$ Abbreviations: BSC, best supportive care; CET, cetuximab. 


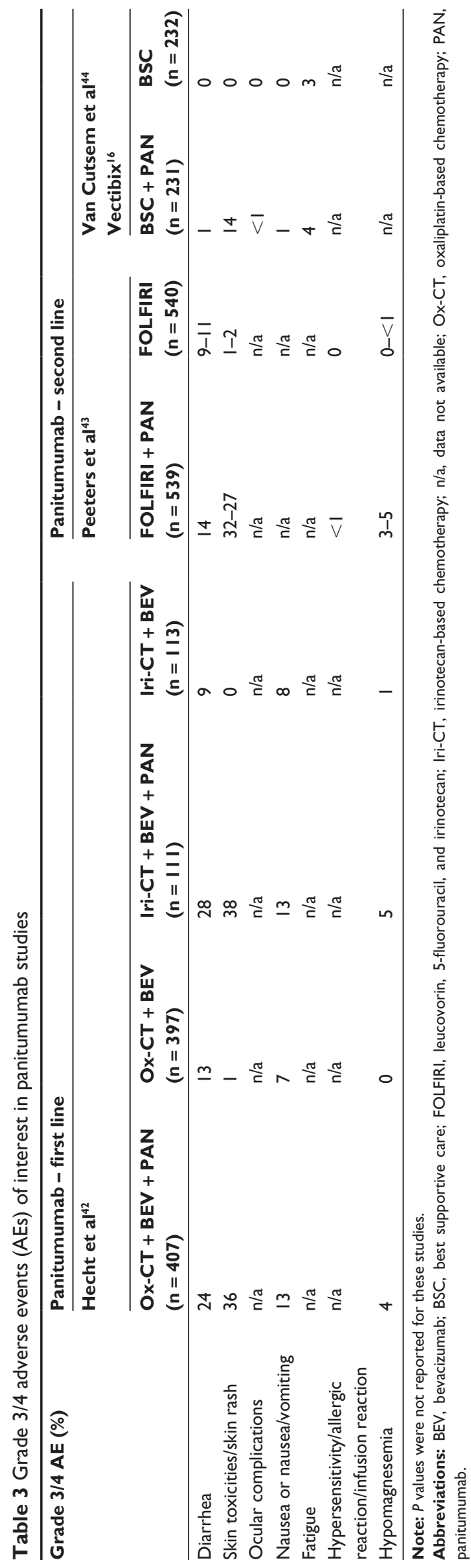

is recommended prior to cetuximab infusions. ${ }^{15}$ In the case of severe infusion reactions, EGFR mAbs should be discontinued. ${ }^{15,16}$

Hypomagnesemia typically develops during treatment with cetuximab or panitumumab, possibly due to EGFR blockade disrupting the active transport of magnesium in the kidneys. ${ }^{50}$ Grade 3 to 4 hypomagnesemia has been observed in $2 \%-6 \%$ of patients receiving cetuximab and in 3\%-5\% of patients receiving panitumumab, compared with $\leq 1 \%$ of patients in the control groups (Tables 2A, B and 3). ${ }^{16,35,40-43,45}$ Intravenous magnesium supplementation has resulted in some success in the treatment of grade 3 or 4 hypomagnesemia in patients receiving cetuximab or panitumumab. ${ }^{51}$

Diarrhea is frequently reported with cetuximab or panitumumab in combination with chemotherapy, as well as with chemotherapy alone. In trials of first- and second-line cetuximab, all-grade diarrhea has been reported in more than $50 \%$ of patients in both the cetuximab and the control treatment groups. ${ }^{35,37,39,40}$ The incidence of grade 3 or 4 diarrhea with cetuximab versus chemotherapy alone was $8 \%-28.4 \%$ versus $6 \%-20 \%$, respectively (Table $2 \mathrm{~A}$ and B). ${ }^{35-40}$ Two of the three studies reporting statistical significance showed significant differences between cetuximab and chemotherapy alone $(P=0.008$ and $P<0.05) ; ;^{36,39}$ in the third study reporting statistical significance, a $P$ value of 0.45 was reported. ${ }^{35} \mathrm{In}$ trials of panitumumab as a combination treatment regimen, the incidence of grade 3 or 4 diarrhea was $24 \%-28 \%$ with first-line panitumumab versus $9 \%-13 \%$ with bevacizumab plus oxaliplatin- or irinotecan-based chemotherapy alone, and $14 \%$ with second-line panitumumab plus FOLFIRI versus $9 \%-11 \%$ with FOLFIRI alone (Table 3 ). ${ }^{42,43}$

The incidence of all-grade nausea has been comparable with cetuximab combined with chemotherapy, compared with chemotherapy alone ( $54 \%-67 \%$ vs $46 \%-73 \%)$. $35,37,39,40$ In trials of cetuximab in first- and second-line settings, the incidence of grade 3 or 4 nausea events with cetuximab compared with the control group was $5 \%$ versus $3 \%$ with FOLFIRI alone, $6 \%$ versus $9 \%$ with XELOX/bevacizumab alone, $14 \%$ versus $7 \%$ with 5 -FU/oxaliplatin alone, $7 \%$ versus $3 \%$ with XELOX alone, and $4.4 \%$ versus $4.3 \%$ with irinotecan alone (Table 2A and B). ${ }^{35,37,39,40}$ There was a similar incidence of grade 3 or 4 nausea with second-line cetuximab plus best supportive care versus best supportive care alone (5.6\% vs 5.5\%) (Table 2B).$^{41}$ In a study of firstline panitumumab in combination with bevacizumab plus oxaliplatin- or irinotecan-based chemotherapy, the incidence of all-grade nausea/vomiting was $71 \%-79 \%$ in the panitumumab treatment groups and $74 \%-75 \%$ in the control 
groups, and the incidence of grade 3 or 4 nausea/vomiting was $\geq 5 \%$ higher with panitumumab versus control $(13 \%$ vs $7 \%-8 \%$ ) (Table 3). ${ }^{42}$ The incidence of grade 3 or 4 nausea reported with second-line panitumumab plus best supportive care was $1 \%$; no grade 3 or 4 nausea was reported with best supportive care alone (Table 3 ). ${ }^{44}$

While phase II and III studies of first-line cetuximab have reported similar rates of grade 3 or 4 fatigue with cetuximab plus chemotherapy compared with FOLFOX4 alone ( $4 \%$ vs $3 \% ; P=0.59$ ) or with FOLFIRI alone (5.3\% vs $4.7 \%$; $P$ value not reported [NR] $),{ }^{36,38}$ a small phase II study reported grade 3 or 4 fatigue in $14 \%$ of patients receiving cetuximab plus XELOX versus 3\% with XELOX alone ( $P$ value NR) (Table 2A). ${ }^{37}$ The incidence of grade 3 or 4 fatigue with cetuximab in the second-line setting was $7.7 \%$ with added cetuximab versus $3.3 \%$ with irinotecan alone $(P<0.05)$, and $33.0 \%$ with cetuximab versus $25.9 \%$ with best supportive care alone $(P=0.09)$ (Table $2 \mathrm{~B}) .{ }^{40,41}$ Grade 3 fatigue was reported in $4 \%$ of patients receiving panitumumab monotherapy compared with $3 \%$ of patients receiving best supportive care alone ( $P$ value NR) (Table 3 ); no grade 4 fatigue was observed. ${ }^{44}$ Fatigue associated with EGFR mAbs should be managed according to general guidelines for this symptom in cancer patients, using a combination of education, counseling on lifestyle strategies, and physical and psychosocial interventions. ${ }^{52}$ In addition, pharmacological interventions, such as psychostimulants, may be considered. ${ }^{52}$

\section{Conclusion}

Currently approved $\mathrm{mAb}$ therapies for the treatment of mCRC that target VEGF or EGFR are associated with consistent AE profiles. Bevacizumab is associated with hypertension, thromboembolism, hemorrhage, and proteinuria, while anti-EGFR mAbs are associated with dermatologic toxicities, hypersensitivity reactions, hypomagnesemia, and ocular complications. Although hypertension and mild bleeding may be managed with standard treatments, discontinuation of bevacizumab may be necessary in some cases. Likewise, discontinuation of EGFR mAbs is necessary with severe hypersensitivity reactions, as well as severe skin toxicities that do not improve with treatment and dose modification. Nausea and diarrhea are common side effects associated with both $\mathrm{mAb}$ agents when they are in combination with chemotherapy; these side effects may be managed with standard supportive care. It also must be emphasized that data collection for AEs can vary across clinical studies, and direct comparisons of incidences reported must be avoided.
Given its role in tumor growth and metastasis, the angiogenesis network remains an attractive target for the development of anticancer therapies. Antiangiogenic therapies that are currently in phase III trials for the treatment of $\mathrm{mCRC}$ include the VEGF Trap, ziv-aflibercept (formerly known as aflibercept); ${ }^{53}$ the antiVEGFR-2 mAb, ramucirumab; ${ }^{54}$ and the tyrosine kinase inhibitors, brivanib alaninate, ${ }^{55}$ cediranib, ${ }^{56,57}$ and regorafenib. ${ }^{58}$ The phase III VELOUR trial of ziv-aflibercept versus placebo in the second-line treatment of $\mathrm{mCRC}$ has ended, and the results have been presented..$^{59}$ In addition, results from a pre-planned interim analysis from the phase III CORRECT trial of regorafenib versus placebo following progression after standard therapies were recently presented at the 2012 Gastrointestinal Cancers Symposium. ${ }^{60}$ Because these agents target VEGF signaling, they have the potential for some of the same on-target vascular toxicities observed with bevacizumab. The results of randomized, phase III trials will be necessary to characterize accurately the safety profiles of these agents and to determine whether overlapping toxicities affect the combination or sequencing of these agents. In addition, there are still unanswered questions regarding the role of chemotherapy in the toxicities observed with antiangiogenic therapies.

\section{Acknowledgments}

Medical editorial writing assistance was provided by Samantha Taylor, PhD, of Phase Five Communications Inc, and supported by sanofi-aventis US LLC, in collaboration with Regeneron Pharmaceuticals.

\section{Disclosure}

The author has no potential conflicts of interest to disclose. The author retained full editorial control over the content of the manuscript and received no compensation from any party for their work.

\section{References}

1. Folkman J. Tumor angiogenesis: therapeutic implications. N Engl J Med. 1971;285(21):1182-1186.

2. Folkman J. Role of angiogenesis in tumor growth and metastasis. Semin Oncol. 2002;29(6 Suppl 16):15-18.

3. Dvorak HF, Brown LF, Detmar M, Dvorak AM. Vascular permeability factor/vascular endothelial growth factor, microvascular hyperpermeability, and angiogenesis. Am J Pathol. 1995;146(5):1029-1039.

4. Ferrara N, Gerber HP, LeCouter J. The biology of VEGF and its receptors. Nat Med. 2003;9(6):669-676.

5. Ferrara N, Kerbel RS. Angiogenesis as a therapeutic target. Nature. 2005;438(7070):967-974.

6. Fischer C, Mazzone M, Jonckx B, Carmeliet P. FLT1 and its ligands VEGFB and PlGF: drug targets for anti-angiogenic therapy? Nat Rev Cancer. 2008;8(12):942-956.

7. Grothey A, Galanis E. Targeting angiogenesis: progress with antiVEGF treatment with large molecules. Nat Rev Clin Oncol. 2009;6(9): $507-518$. 
8. André T, Kotelevets L, Vaillant JC, et al. Vegf, Vegf-B, Vegf-C and their receptors KDR, FLT-1 and FLT-4 during the neoplastic progression of human colonic mucosa. Int J Cancer. 2000;86(2):174-181.

9. Zhang F, Tang Z, Hou X, et al. VEGF-B is dispensable for blood vessel growth but critical for their survival, and VEGF-B targeting inhibits pathological angiogenesis. Proc Natl Acad Sci U S A. 2009;106(15):6152-6157.

10. Bates RC, Goldsmith JD, Bachelder RE, et al. Flt-1-dependent survival characterizes the epithelial-mesenchymal transition of colonic organoids. Curr Biol. 2003;13(19):1721-1727.

11. Odorisio T, Schietroma C, Zaccaria ML, et al. Mice overexpressing placenta growth factor exhibit increased vascularization and vessel permeability. J Cell Sci. 2002;115(Pt 12):2559-2567.

12. Avastin (bevacizumab) [prescribing information]. South San Francisco, CA: Genentech; 2011.

13. Presta LG, Chen H, O'Connor SJ, et al. Humanization of an anti-vascular endothelial growth factor monoclonal antibody for the therapy of solid tumors and other disorders. Cancer Res. 1997;57(20):4593-4599.

14. European Medicines Agency. Avastin summary of product characteristics. Available at: http://www.ema.europa.eu/ema/index.jsp?curl=pages/medi cines/human/medicines/000582/human_med_000663.jsp\&murl=menus/ medicines/medicines.jsp\&mid=WC0b01ac058001d124. Accessed October 8, 2011.

15. Erbitux (cetuximab) [prescribing information]. Princeton, NJ: BristolMyers Squibb Company; 2011.

16. Vectibix (panitumumab) [prescribing information]. Thousand Oaks, CA: Amgen Inc.; 2011.

17. Perrotte $\mathrm{P}$, Matsumoto $\mathrm{T}$, Inoue $\mathrm{K}$, et al. Anti-epidermal growth factor receptor antibody $\mathrm{C} 225$ inhibits angiogenesis in human transitional cell carcinoma growing orthotopically in nude mice. Clin Cancer Res. 1999;5(2):257-265.

18. Spano JP, Lagorce C, Atlan D, et al. Impact of EGFR expression on colorectal cancer patient prognosis and survival. Ann Oncol. 2005;16(1) 102-108.

19. European Medicines Agency. Erbitux summary of product characteristics. Available at: http://www.ema.europa.eu/ema/index. jsp?curl=pages $/$ medicines $/$ human $/$ medicines $/ 000558 /$ human med_000769.jsp\&jsenabled=true. Accessed October 8, 2011.

20. European Medicines Agency. Vectibix summary of product characteristics. Available at: http://www.ema.europa.eu/ema/index. jsp?curl=pages $/$ medicines $/$ human/medicines $/ 000741 /$ human med_001128.jsp\&mid=WC0b01ac058001d124. Accessed October 8, 2011.

21. European Medicines Agency. Vectibix summary of opinion. Available at: http://www.ema.europa.eu/ema/index.jsp?curl=pages/medicines/ human/medicines/000741/smops/Positive/human_smop_000203. jsp\&mid=WC0b01ac058001d127. Accessed October 8, 2011.

22. Hurwitz H, Fehrenbacher L, Novotny W, et al. Bevacizumab plus irinotecan, fluorouracil, and leucovorin for metastatic colorectal cancer N Engl J Med. 2004;350(23):2335-2342.

23. Kabbinavar F, Hurwitz HI, Fehrenbacher L, et al. Phase II, randomized trial comparing bevacizumab plus fluorouracil (FU)/leucovorin (LV) with $\mathrm{FU} / \mathrm{LV}$ alone in patients with metastatic colorectal cancer. $J$ Clin Oncol. 2003;21(1):60-65.

24. Kabbinavar FF, Schulz J, McCleod M, et al. Addition of bevacizumab to bolus fluorouracil and leucovorin in first-line metastatic colorectal cancer: results of a randomized phase II trial. J Clin Oncol. 2005;23(16):3697-3705.

25. Saltz LB, Clarke S, Díaz-Rubio E, et al. Bevacizumab in combination with oxaliplatin-based chemotherapy as first-line therapy in metastatic colorectal cancer: a randomized phase III study. J Clin Oncol. 2008;26(12):2013-2019.

26. Giantonio BJ, Catalano PJ, Meropol NJ, et al. Bevacizumab in combination with oxaliplatin, fluorouracil, and leucovorin (FOLFOX4) for previously treated metastatic colorectal cancer: results from the Eastern Cooperative Oncology Group Study E3200. J Clin Oncol. 2007;25(12):1539-1544.
27. Wagner AD, Arnold D, Grothey AA, Haerting J, Unverzagt S. Antiangiogenic therapies for metastatic colorectal cancer. Cochrane Database Syst Rev. 2009;(3):CD005392.

28. Izzedine H, Ederhy S, Goldwasser F, et al. Management of hypertension in angiogenesis inhibitor-treated patients. Ann Oncol. 2009;20(5): $807-815$.

29. Scappaticci FA, Skillings JR, Holden SN, et al. Arterial thromboembolic events in patients with metastatic carcinoma treated with chemotherapy and bevacizumab. $J$ Natl Cancer Inst. 2007;99(16): 1232-1239.

30. Ranpura V, Hapani S, Chuang J, Wu S. Risk of cardiac ischemia and arterial thromboembolic events with the angiogenesis inhibitor bevacizumab in cancer patients: a meta-analysis of randomized controlled trials. Acta Oncol. 2010;49(3):287-297.

31. Schutz FA, Je Y, Azzi GR, Nguyen PL, Choueiri TK. Bevacizumab increases the risk of arterial ischemia: a large study in cancer patients with a focus on different subgroup outcomes. Ann Oncol. 2011;22(6): 1404-1412.

32. Saif MW. Managing bevacizumab-related toxicities in patients with colorectal cancer. J Support Oncol. 2009;7(6):245-251.

33. Keefe D, Bowen J, Gibson R, Tan T, Okera M, Stringer A. Noncardiac vascular toxicities of vascular endothelial growth factor inhibitors in advanced cancer: a review. Oncologist. 2011;16(4):432-444.

34. Tol J, Cats A, Mol L, et al. Gastrointestinal ulceration as a possible side effect of bevacizumab which may herald perforation. Invest New Drugs. 2008;26(4):393-397.

35. Tol J, Koopman M, Rodenburg CJ, et al. A randomised phase III study on capecitabine, oxaliplatin and bevacizumab with or without cetuximab in first-line advanced colorectal cancer, the CAIRO2 study of the Dutch Colorectal Cancer Group (DCCG). An interim analysis of toxicity. Ann Oncol. 2008;19(4):734-738.

36. Van Cutsem E, Köhne CH, Hitre E, et al. Cetuximab and chemotherapy as initial treatment for metastatic colorectal cancer. $N$ Engl J Med. 2009;360(14):1408-1417.

37. Borner M, Koeberle D, Von Moos R, et al. Adding cetuximab to capecitabine plus oxaliplatin (XELOX) in first-line treatment of metastatic colorectal cancer: a randomized phase II trial of the Swiss Group for Clinical Cancer Research SAKK. Ann Oncol. 2008;19(7): $1288-1292$.

38. Bokemeyer C, Bondarenko I, Makhson A, et al. Fluorouracil, leucovorin, and oxaliplatin with and without cetuximab in the first-line treatment of metastatic colorectal cancer. J Clin Oncol. 2009;27(5): 663-671.

39. Adams RA, Meade AM, Madi A, et al. Toxicity associated with combination oxaliplatin plus fluoropyrimidine with or without cetuximab in the MRC COIN trial experience. Br J Cancer. 2009;100(2): 251-258.

40. Sobrero AF, Maurel J, Fehrenbacher L, et al. EPIC: phase III trial of cetuximab plus irinotecan after fluoropyrimidine and oxaliplatin failure in patients with metastatic colorectal cancer. J Clin Oncol. 2008;26(14):2311-2319.

41. Jonker DJ, O'Callaghan CJ, Karapetis CS, et al. Cetuximab for the treatment of colorectal cancer. $N$ Engl J Med. 2007;357(20): 2040-2048.

42. Hecht JR, Mitchell E, Chidiac T, et al. A randomized phase IIIB trial of chemotherapy, bevacizumab, and panitumumab compared with chemotherapy and bevacizumab alone for metastatic colorectal cancer. J Clin Oncol. 2009;27(5):672-680.

43. Peeters M, Price TJ, Cervantes A, et al. Randomized phase III study of panitumumab with fluorouracil, leucovorin, and irinotecan (FOLFIRI) compared with FOLFIRI alone as second-line treatment in patients with metastatic colorectal cancer. J Clin Oncol. 2010;28(31): 4706-4713.

44. Van Cutsem E, Peeters M, Siena S, et al. Open-label phase III trial of panitumumab plus best supportive care compared with best supportive care alone in patients with chemotherapy-refractory metastatic colorectal cancer. J Clin Oncol. 2007;25(13):1658-1664. 
45. Van Cutsem E, Siena S, Humblet Y, et al. An open-label, single-arm study assessing safety and efficacy of panitumumab in patients with metastatic colorectal cancer refractory to standard chemotherapy. Ann Oncol. 2008;19(1):92-98.

46. Lacouture ME, Mitchell EP, Piperdi B, et al. Skin toxicity evaluation protocol with panitumumab (STEPP), a phase II, open-label, randomized trial evaluating the impact of a pre-emptive skin treatment regimen on skin toxicities and quality of life in patients with metastatic colorectal cancer. J Clin Oncol. 2010;28(8):1351-1357.

47. Saltz LB, Meropol NJ, Loehrer PJ Sr, Needle MN, Kopit J, Mayer RJ. Phase II trial of cetuximab in patients with refractory colorectal cancer that expresses the epidermal growth factor receptor. $J$ Clin Oncol. 2004;22(7):1201-1208.

48. Burtness B, Anadkat M, Basti S, et al. NCCN Task Force report: management of dermatologic and other toxicities associated with EGFR inhibition in patients with cancer. J Natl Compr Cancer Netw. 2009;7(Suppl 1):S5-S21.

49. Chung $\mathrm{CH}$, Mirakhur B, Chan E, et al. Cetuximab-induced anaphylaxis and IgE specific for galactose-alpha-1,3-galactose. $N$ Engl J Med. 2008;358(11):1109-1117.

50. Schrag D, Chung KY, Flombaum C, Saltz L. Cetuximab therapy and symptomatic hypomagnesemia. J Natl Cancer Inst. 2005;97(16): 1221-1224.

51. Fakih M. Management of anti-EGFR-targeting monoclonal antibodyinduced hypomagnesemia. Oncology (Williston Park). 2008;22(1): 74-76.

52. National Comprehensive Cancer Network (NCCN) Clinical Practice Guidelines in Oncology (NCCN Guidelines ${ }^{\sqrt{\mathbb{R}}}$ ). Cancer-Related Fatigue. Version 1.2012. Available from: http://www.nccn.org. Accessed March 1, 2012.

53. Sanofi-Aventis; Regeneron Pharmaceuticals; National Surgical Adjuvant Breast and Bowel Project (NSABP). Aflibercept Versus Placebo in Combination With Irinotecan and 5-FU in the Treatment of Patients With Metastatic Colorectal Cancer After Failure of an Oxaliplatin Based Regimen (VELOUR). In: ClinicalTrials.gov [Internet]. Bethesda (MD): National Library of Medicine (US). 2000- [cited 2011 Oct 8]. Available from: http://clinicaltrials.gov/ct2/show/NCT00561470 NLM Identifier: NCT00561470.
54. Eli Lilly and Company; ImClone LLC. A Study in Second Line Metastatic Colorectal Cancer. In: ClinicalTrials.gov [Internet]. Bethesda (MD): National Library of Medicine (US). 2000- [cited 2011 Oct 8]. Available from: http://clinicaltrials.gov/ct2/show/NCT01183780 NLM Identifier: NCT01183780.

55. NCIC Clinical Trials Group. Cetuximab With or Without Brivanib in Treating Patients With K-Ras Wild Type Tumours and Metastatic Colorectal Cancer. In: ClinicalTrials.gov [Internet]. Bethesda (MD): National Library of Medicine (US). 2000- [cited 2011 Oct 8]. Available from: http://clinicaltrials.gov/ct2/show/NCT00640471 NLM Identifier: NCT00640471.

56. AstraZeneca. First Line Metastatic Colorectal Cancer Therapy in Combination With FOLFOX (HORIZON III). In: ClinicalTrials.gov [Internet]. Bethesda (MD): National Library of Medicine (US). 2000[cited 2011 Oct 8]. Available from: http://clinicaltrials.gov/ct2/show/ NCT00384176 NLM Identifier: NCT00384176.

57. AstraZeneca. Cediranib (AZD2171, RECENTIN ${ }^{\mathrm{TM}}$ ) in Addition to Chemotherapy in Patients With Untreated Metastatic Colorectal Cancer (HORIZON II). In: ClinicalTrials.gov [Internet]. Bethesda (MD): National Library of Medicine (US). 2000- [cited 2011 Oct 8]. Available from: http://clinicaltrials.gov/ct2/show/NCT00399035 NLM Identifier: NCT00399035.

58. Bayer. Patients With Metastatic Colorectal Cancer Treated With Regorafenib or Placebo After Failure of Standard Therapy. In: ClinicalTrials. gov [Internet]. Bethesda (MD): National Library of Medicine (US). 2000- [cited 2011 Oct 8]. Available from: http://clinicaltrials.gov/ct2/ show/NCT01103323 NLM Identifier: NCT01103323.

59. Van Cutsem E, Tabernero J, Lakomy R, et al. Intravenous (IV) aflibercept versus placebo in combination with irinotecan/5-FU (FOLFIRI) for second-line treatment of metastatic colorectal cancer (MCRC): results of a multinational phase III trial (EFC10262-VELOUR). Ann Oncol. 2011;22 (Suppl 5):Abstract O-0024.

60. Grothey A, Sobrero AF, Siena S, et al. Results of a phase III randomized, double-blind, placebo-controlled, multicenter trial (CORRECT) of regorafenib plus best supportive care (BSC) versus placebo plus BSC in patients (pts) with metastatic colorectal cancer (mCRC) who have progressed after standard therapies. J Clin Oncol. 2010;30 (Suppl 4): Abstract LBA38.
OncoTargets and Therapy

\section{Publish your work in this journal}

OncoTargets and Therapy is an international, peer-reviewed, open access journal focusing on the pathological basis of all cancers, potential targets for therapy and treatment protocols employed to improve the management of cancer patients. The journal also focuses on the impact of management programs and new therapeutic agents and protocols on

\section{Dovepress}

patient perspectives such as quality of life, adherence and satisfaction The manuscript management system is completely online and includes a very quick and fair peer-review system, which is all easy to use. Visit http://www.dovepress.com/testimonials.php to read real quotes from published authors. 